\title{
Koch's Abdomen- Successful Management of Abdominal Cocoon with Jejunostomy and Second Line Antitubercular Drugs
}

\author{
Dr. Saurabh Tiwari ${ }^{1 *}$, Dr. Shahaji Deshmukh ${ }^{2}$, Dr. Vishesh Dikshit ${ }^{3}$, Dr. Abhaya Gupta ${ }^{4}$, Dr. Paras Kothari ${ }^{5}$
}

${ }^{1}$ MCH Pediatric Surgery, 3year Resident, ${ }^{2}$ Assistant Professor, ${ }^{3}$ Associate Professor, ${ }^{4}$ Associate Professor, ${ }^{5}$ Professor and Head of Department, Department of Pediatric Surgery, LTMMC \& GH, Sion, Mumbai, India

DOI: $10.36347 /$ sjams.2020.v08i07.001

| Received: 22.06.2020 | Accepted: 29.06.2020 | Published: 04.07.2020

*Corresponding author: Dr. Saurabh Tiwari

Abstract

Abdominal tuberculosis (ATB) in children poses a diagnostic challenge because of its nonspecific clinical features, often delaying the diagnosis. The annual incidence of tuberculosis (TB) is nearly 10.1 million, including around 1 million children [1]. It is estimated that worldwide, 239000 children younger than 15 years die from TB in a year [2]. TB being endemic in India accounts for about a quarter of the global TB cases, the highest in the world [3]. We hereby report a case of intestinal obstruction in a 6 month old child secondary to abdominal koch's. The child underwent jejunostomy for severe bowel adhesions, frozen pelvis and cocoon formation. Histopathology of omentum and mesenteric lymphnodes confirmed it to be abdominal tuberculosis. With parenteral nutrition and injectable antitubercular drugs(second line) for 7 weeks before stoma closure and 2 weeks later patient could be managed successfully and discharged on oral ATT(Anti tubercular treatment).We could successfully manage abdominal tuberculosis with jejunostomy(high output fistula) with second line injectable ATT and total parenteral nutrition.

Keywords: Abdominal tuberculosis, children, jejunostomy, laparotomy, mycobacterium.

Copyright @ 2020: This is an open-access article distributed under the terms of the Creative Commons Attribution license which permits unrestricted use, distribution, and reproduction in any medium for non-commercial use (NonCommercial, or CC-BY-NC) provided the original author and source are credited.

\section{INTRODUCTION}

Almost $10 \%$ of the cases of TB in India are of pediatric TB (tuberculosis), with pulmonary involvement being the most common site of involvement [4]. Overall, abdominal TB (ATB) is the sixth most frequent extrapulmonary site after lymphatic, genitourinary, bone and joint, miliary and meningeal tuberculosis [5]. It is an uncommon presentation of TB, seen in only $0.3 \%$ of pediatric TB. It may involve the gastrointestinal (GI) tract, peritoneum, lymph nodes, or solid viscera (liver, spleen, and pancreas). Most common causative organism of abdominal tuberculosis is Mycobacterium tuberculosis with $\mathrm{M}$. bovis and Mycobacterium avium complex in immunocompromised or HIV/AIDS coinfection patients [6].

\section{Case Report}

6 month old female was brought with complaints of loose stools, abdominal distension since 2 months and history of fever since 2 weeks. There was history of weight loss. There was no history of NICU stay. The mother was diagnosed with central nervous system tuberculosis and was on ATT for 3 months. Child was moderately pale and malnourished. Abdomen was soft, moderately distended with palpable bowel loops but no signs of peritonitis. Per rectal examination had no mass, bleeding with stools present.

Radiograph of erect abdomen showed dilated bowel loops with gas upto pelvis. (Figure 1). Abdominal ultrasound showed circumferential thickening of distal ileal loop, omental thickening with fat stranding and multiple enlared necrotic and nonnecrotic lymph nodes with mild ascites.

Computed tomography scan of abdomen had bowel wall thickening with peritoneal and omental nodularity. Multiple conglomerated necrotic \& non necrotic pre, para aortic and peripancreatic lymph nodes and moderate free fluid in abdomen was present (Figure 2 ). Features were highly suggestive of abdominal tuberculosis.

Colonoscopy was normal and the patient was started on CATEGORY 1 ATT. Patient had increasing abdominal distension and increasing amount of Ryle's tube aspirates and hence was taken up for surgery. There were dense interbowel adhesions, cocoon formation, frozen pelvis with caseation of mesenteric lymph nodes with tubercles over omentum (Figure 3). Small bowel from duodenojejunal(DJ) flexure to distal jejunum was free and beyond that there was severe 
clumping of bowel loops. Loop Jejunostomy was done $40 \mathrm{~cm}$ from DJ flexure with tension band suturing. Histopathology of omentum and mesenteric lymph nodes was conclusive of abdominal tuberculosis with gene Xpert detecting mycobacteria with no rifampicin resistance.Total parenteral nutrition was initiated. Injectable second line ATT was given for 7 weeks prior to stoma closure in the form of levofloxacin $(10 \mathrm{mg} / \mathrm{kg}$ once daily), amikacin (15 $\mathrm{mg} / \mathrm{kg}$ once daily), azithromycin $(15 \mathrm{mg} / \mathrm{kg}$ twice daily) and linezolid (10 $\mathrm{mg} / \mathrm{kg}$ twice daily) after consultation with chest and tuberculosis department.Nestum rice was instilled via distal stoma 1 month after surgery to confirm distal bowel patency. Gradually patient started passing nestum rice per rectally with no evidence of any web, obstruction or perforation. Child was planned for stoma closure and a distal stoma dye study showed dye passing per rectally and continuity of bowel maintained (Figure 4).

After stoma closure injectable ATT was given for 1 week and another week of overlapping of injectable ATT with oral ATT. Patient was then discharged on oral ATT. At one month follow up patient doing fine with adequate weight gain.

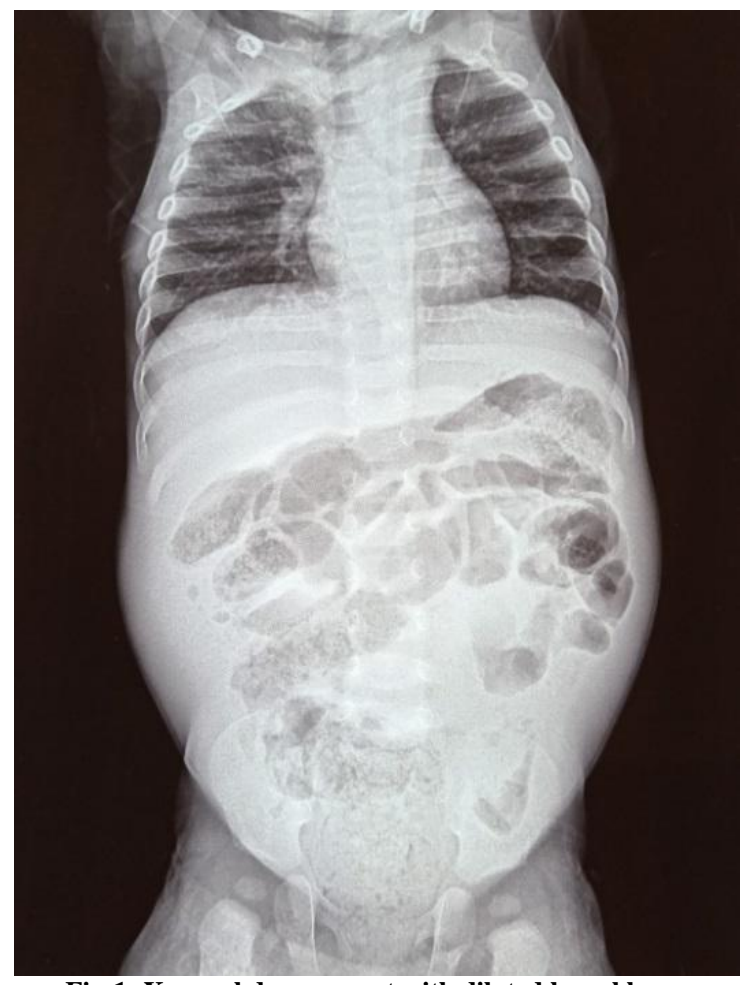

Fig-1: X-ray abdomen erect with dilated bowel loops

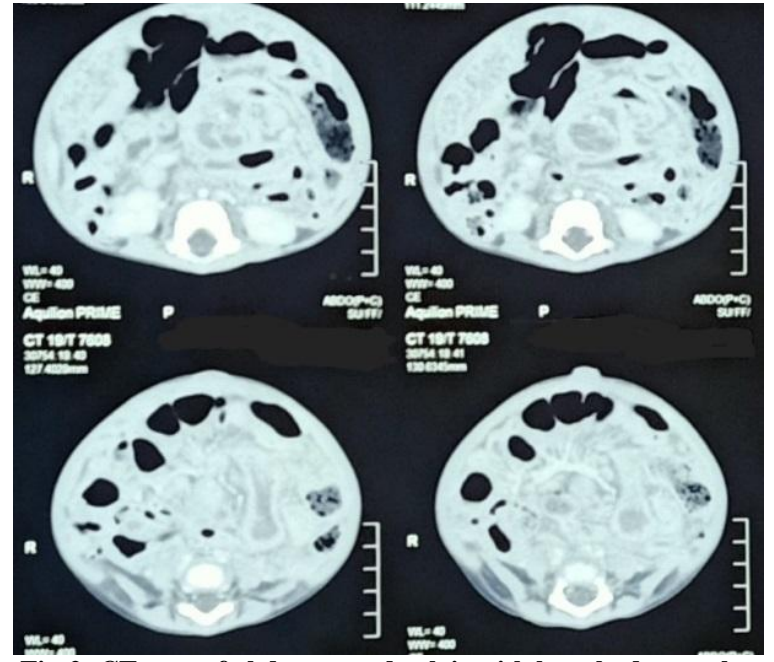

Fig-2: CT scan of abdomen and pelvis with lymphadenopathy, ascites, bowel and omental thickening

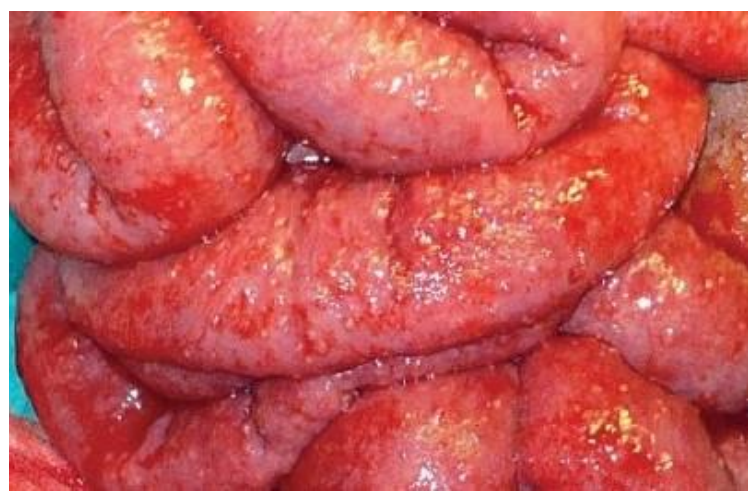

Fig-3: Intraoperative photograph showing bowel adhesions, thickening and tubercles

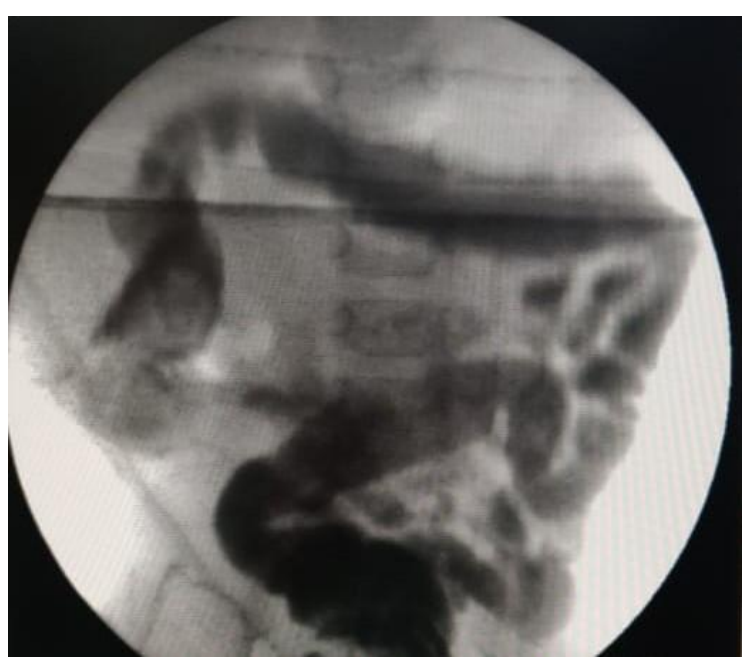

Fig-4: Distal stoma dye study showing distal bowel patency 


\section{DISCUSSION}

Chronic abdominal pain, palpable abdominal lump, with other non-specific manifestations makes an early diagnosis difficult. Clinicians usually start antitubercular therapy in microbiologically confirmed or clinically diagnosed cases of tuberculosis. The modes of infection for intestinal tuberculosis include hematogenous spread from a primary lung focus, spread via lymphatics from infected nodes, ingestion of bacilli either from the sputum or from infected sources such as milk products, or by direct spread from adjacent organs. It usually presents late with complications especially acute or sub-acute intestinal obstruction due to mass (tuberculoma) or stricture formation in small bowel and ileocaecal region or gut perforation leading to peritonitis. Most common complication of abdominal tuberculosis is obstruction due to narrowing of the lumen by hyperplastic caecal tuberculosis, by strictures of the small intestine, which are commonly multiple, or by adhesions for which emergency surgery has to be resorted to. Our patient had adhesions with tubercles all over abdomen.

Most common site of involvement of intestinal TB is the ileocaecal region, possibly because of the increased physiological stasis, increased rate of fluid and electrolyte absorption, minimal digestive activity and an abundance of lymphoid tissue at this site

\section{ATB Is divided into four patterns of involvement}

1. Peritoneal-including wet type (characterized by ascites formation), dry fibrotic type (associated with peritoneal thickening, adhesions, omental, and mesenteric thickening with little or no ascites), mixed type (combination of both), and abdominal cocoon (characterized by presence of a membranous sac around the intestinal loops).

2. Gastrointestinal tract-involvement as found on imaging and/or endoscopy and/or histology

3. Visceral organs-Focal granulomas, generally multiple may be found in liver, pancreas or spleen

4. Abdominal lymph nodes-Mesentric and retroperitoneal lymph nodes when affected may get caseated and calcify later.

Microbiological yield is low, and one often has to start therapy based on a strong clinical suspicion. A close follow up for the resolution of clinical and imaging features is warranted.

In the chronic form of abdominal tuberculosis, fever, pain, altered bowel habits, anorexia, weight loss and malaise are the most common symptoms of abdominal tuberculosis. Bowel perforation or acute intestinal obstruction are the acute presentations of abdominal tuberculosis.
Specific results are obtained from tissue and other infected material samples on microbiological/histopathological/genetic analysis methods. The surgeon plays a vital role by making a clinical diagnosis based upon the intra-operative findings despite negative tissue sample results and patient gaining relief on commencement of ATT.

Predictors of need for surgery are long strictures $(>12 \mathrm{~cm})$ and multiple areas of involvement. According to Balasubramanian et al.[7] the mean time interval required for the relief of obstructive symptoms was 6 months from initiation of drug therapy, hence ATT is preferred for 9-12 months.

Intestinal obstruction due to varied manifestations of abdominal Koch's are the commonest etiology for surgical intervention, followed closely by bowel perforation, intraperitoneal abscess, etc

\section{Conclusion}

Adequate, intensive and timely surgical intervention/laparotomy, with nutritional support, stoma care, management of sepsis, better transport \& referral system in remote places and concurrent ATT can result in prompt relief and speedy recovery of patients with abdominal tuberculosis. We hereby conclude that successful management is achievable with second line ATT and total parenteral nutrition in the presence of high output fistula.

\section{REFERENCES}

1. GBD Tuberculosis Collaborators. The global burden of tuberculosis: results from the Global Burden of Disease Study 2015.Lancet Infect.Dis.2018;18: 261-84.

2. Dodd PJ, Yuen CM, Sismanidis C, Seddon JA, Jenkins HE. The global burden of tuberculosis mortality in children: a mathematical modelling study.Lancet Glob. Health. 2017; 5: e898-906.

3. World Health Organization. Global Tuberculosis Report 2016. Geneva: The Organization, 2016;1192. Cited 20 May 2019. Available from URL: https://apps.who.int/medicinedocs/en/d/Js23098en/

4. Mandal A, Singh A. Recent changes in tuberculosis guidelines for children.Mycobact. Dis.2017;7: 237.

5. Sharma MP, Bhatia V. Abdominal tuberculosis.Indian J. Med. Res.2004;120: 305-15

6. Tuberculosis in India. Available at: https://en.wikipedia.org/wiki/Tuberculosis_in_Indi a. Accessed on 31 March 2019.

7. Balasubramanian R, Ramachandran R, Joseph PE, Nagarajan M, Thiruvengadam KV, Tripathy SP. Interim results of a clinical study of abdominal tuberculosis. Indian J Tuberc. 1989;36:117-21. 